\title{
Kooperation zwischen nationalen und europäischen Gerichten
}

\author{
Von Ferdinand Kirchhof, Karlsruhe*
}

Zwei europäische Gerichte und in den Ländern oft zwei nationale Gerichte kümmern sich um die Rechte der Bürger. Ihnen stehen dabei vier Grundrechtstexte zur Seite: die Menschenrechtskonvention, die europäische Grundrechtecharta sowie die Verfassungen der Mitgliedstaaten und ihrer Länder.

Diese Redundanz summiert Schutzvorschriften, optimiert aber oftmals nicht den Schutz des Bürgers. Um zu einem wirksamen Grundrechtsschutz zu gelangen, sind die Kompetenzschranken der Gerichte zu beachten; vor allem die Menschenrechtskonvention und Art. $51 \mathrm{ff}$. EuGRCh begrenzen die Tätigkeit europäischer Vertragsgerichte. Darüber hinaus sind gerichtliche Zurückhaltung und Dialog zwischen den Gerichten notwendig, um den Grundrechtsschutz tatsächlich zu optimieren.

\section{Optimierung oder Atomisierung des Grundrechtsschutzes? - Das Problem der Fülle von Grundrechten und der Vielzahl von Verfassungsgerichten -}

Alle europäischen Staaten hat in den letzten 20 Jahren ein Verfassungsbewusstsein erfasst. Es ist mittlerweile selbstverständlich, dass jedem Menschen Grundrechte zustehen, die vom und gegen den Staat zu schützen sind. Mittlerweile gibt es einen Kanon von allgemein und umfassend anerkannten Menschenrechten, die europaweit und national gelten. Zu ihrem Schutze stehen in den meisten Staaten Verfassungsgerichte bereit; daneben bemühen sich der Europäische Gerichtshof um die Gewährleistung der Europäischen Grundrechtecharta in den 28 Mitgliedstaaten der EU und der Europäische Gerichtshof für Menschenrechte um die Einhaltung der Europäischen Menschenrechtskonvention in den 47 Mitgliedstaaten des Europarats. In bundesstaatlich aufgebauten Ländern sind zu diesem Zweck meist mehrere Verfassungsgerichte, nämlich auf der Bundesebene und in den Ländern dieses Staates, vorhanden. So weist zum Beispiel die Bundesrepublik Deutschland auf nationaler Ebene ein Bundesverfassungsgericht auf, das das Grundgesetz als Bundesverfassung sichert, und daneben in allen 16 Ländern ein eigenes Landesverfassungsgericht zum Schutz der Grundrechte in den Landesverfassungen. Im Maximalfall können sich also vier Gerichte um die Grundrechte des Bürgers kümmern und vier Normenkomplexe für seinen umfassenden Schutz sorgen.

Jede der vier Grundrechtskataloge kann identische Schutzgewährungen, Verbürgungen bloß ähnlicher Schutzrichtung unterschiedlichen Tatbestands oder differenter Eingriffserlaubnisse und auch besondere, regionale Schutzrechte enthalten. Gewährleistungen mit identischem Schutzziel existieren zum Beispiel häufig bei

* Prof. Dr. Ferdinand Kirchhof ist Vizepräsident des Bundesverfassungsgerichts, Vorsitzender des Ersten Senats und Inhaber eines Lehrstuhls für Öffentliches Recht, Finanz- und Steuerrecht an der Eberhard Karls Universität Tübingen. Zudem hat Ferdinand Kirchhof einen Jean-Monnet-Chair for European Fiscal Law inne. 
den Grundrechten auf Eigentum ${ }^{1}$ oder Beruf ${ }^{2}$. Hier zielen alle Normkomplexe in die identische Richtung und ziehen alle Verfassungsgerichte am selben Strang. Das wirft die Frage auf, wie vier Verfassungsgerichte im konkreten Fall den Bürger wirksam schützen. Das Bemühen von vier gesetzgebenden Organen, dem Bürger Grundrechte zuzusprechen, erweist sich als gut gemeinte Tat, die nicht immer zur Optimierung des Grundrechtsschutzes beiträgt, denn unterschiedliche Spruchkörper führen unvermeidlich zu differenter Rechtsprechung und drängen so den identisch gewollten Grundrechtsschutz in unterschiedliche Richtungen. ${ }^{3}$ Disparater Grundrechtsschutz ist die Folge, ${ }^{4}$ taktisches Kalkül bei der Auswahl zwischen vier Gerichten liegt für den kundigen Juristen nahe.

Dringlicher wird eine Problemlösung, wenn für denselben Sachverhalt auf zwei oder mehreren Ebenen Grundrechte anzuwenden sind, die in dieselbe Richtung zielen, aber differente Normtexte aufweisen. So können die Schutzgüter von ähnlichen Grundrechten differieren, wenn zum Beispiel das Recht auf Eigentum im einen Staat nur körperliche Gegenstände umfasst, im anderen immaterielle Urheber-, Markenund Patentrechte mit einbezieht und im dritten Staat sogar Renten und Sozialversicherungsansprüche gewährleistet. ${ }^{5}$

Noch mehr Probleme bereiten unterschiedliche Rechtfertigungsgründe für einen Eingriff in identische Schutzgüter. Der eine Staat kann zum Beispiel die Beschränkung des Berufs nur aus Gründen der öffentlichen Sicherheit und Ordnung, der andere auch zur Wirtschaftslenkung oder ein dritter sogar zum Schutz aller einfachgesetzlichen Rechtsgüter ${ }^{6}$ erlauben.

Vor allem in mehrpoligen Grundrechtsverhältnissen sind es sogar die Schutzziele der daran beteiligten Grundrechte selbst, welche in Konkordanz zu bringen sind und damit differente Beschränkungsmöglichkeiten ergeben. Weitere Schwierigkeiten entstehen durch einen unterschiedlichen Geltungsbereich von Grundrechten. Schützen sie wie in Deutschland nur den Bürger vor der Staatsgewalt, ${ }^{7}$ wird der gesamte Privatrechtsverkehr zwischen Bürgern und Wirtschaftssubjekten ausgespart. Wird ihre Verbindlichkeit hingegen auf Rechtsbeziehungen zwischen Privaten erweitert $^{8}$, kommt es zu einer völlig anderen Rechtsprechung.

1 Art. 14 Abs. 1 GG, Art. 1 Abs. 1 EMRK-ZP 1, Art. 17 Abs. 1 GRCh, und beispielsweise Art. 45 HessVerf.

2 Art. 12 Abs. 1 GG, Art. 15, 16 GRCh und etwa Art. 151 Abs. 2, Art. 166 BayVerf. Die EMRK enthält zwar keine Gewährleistung der Berufsfreiheit, der EGMR neigt aber zu einer extensiven Interpretation der Eigentumsgarantie. Vgl. D. Richter, in: Grote/Marauhn (Hrsg.), EMRK/GG, 2006, Kap. 9, Rn. 41.

3 Vgl. „Caroline v. Hannover“: BVerfGE 97, 125; 101, 361; 120, 180; EGMR, Urteil v. 24.6.2004 - 59320/00, NJW 2004, S. 2647; „Görgülü“: BVerfGE 111, 307; EGMR, Urteil v. 26.2.2004 - 74969/01, NJW 2004, S. 3397, Rn. 37, 26; „Sicherungsverwahrung“: BVerfGE 109, 133; 128, 326; EGMR, Urteil v. 17.12.2009 - 19359/04, NJW 2010, S. 2495.

4 Vgl. F. Kirchhof, Grundrechtsschutz durch europäische und nationale Gerichte, NJW 2011, S. 3681 f.; P. M. Huber, Auslegung und Anwendung der Charta der Grundrechte, NJW 2011, S. 2385.

5 So etwa in Deutschland, vgl. BVerfGE 31, 229; 49, 382 ff.; 53, 257, 289 ff.; 76, 256, 293.

6 Auf der Stufe der Berufsausübungsregelungen können in Deutschland bereits ,,vernünftige Erwägungen des Gemeinwohls" einen Eingriff rechtfertigen, BVerfGE 7, $377 \mathrm{ff}$.

7 H.-J. Papier, in: Merten/ders. (Hrsg.), Handbuch der Grundrechte, Band II, 2006, § 55, Rn. $23 \mathrm{ff}$.

8 Zur Drittwirkung der GRCh, P.M. Huber (Fn. 4), S. 2389 f. 
Wo auf zwei oder mehr Ebenen identische oder ähnliche Grundrechte gelten, muss ein Mechanismus Kollisionen verhindern. Entweder sind die Anwendungsbereiche der Normen und die Kompetenzen der Gerichte fest gegeneinander abzugrenzen oder es muss die Rechtsprechung zwischen den Ebenen einander angepasst werden. Ein unabgestimmtes „Gegeneinander-Judizieren“ von Gerichten würde den Grundrechten schaden statt sie zu schützen.

\section{Die Methode strikter Kompetenzabgrenzung}

Am einfachsten ist es, die Kontrollbefugnisse der Gerichte strikt und ohne Grauzonen voneinander abzugrenzen. Diese Vorgehensweise hat sich für das Verhältnis zwischen Verfassungsgerichten eines föderal organisierten Staates bewährt. Die auf dem pouvoir constituant des Volkes eines souveränen Staates entspringende nationale Verfassung ordnet die Bereiche von Bund und Ländern dann meist in der Weise, dass die bundesstaatlichen Grundrechte und die Entscheidungen des Verfassungsgerichts des Bundes Vorrang genießen. ${ }^{9}$ Kollisionen zwischen nationalen Verfassungsnormen und eine disparate Rechtsprechung werden auf diese Weise im Staat ausgeschlossen. Hier ist das Problem vollständig und zufriedenstellend gelöst.

Schwieriger erweist es sich aber, die Rechtsprechung der nationalen Verfassungsgerichte, des Europäischen Gerichtshofs und des Europäischen Gerichtshofs für Menschenrechte aufeinander abzustimmen. Die Technik aufeinander abgestimmter Kompetenzzuweisungen ist hier zwar möglich und wird auch eingesetzt. Sie stößt aber an Grenzen, wo im konkreten Fall die Aktionsebenen verschränkt sind, denn dann würde ein Grundrechtskomplex regelmäßig völlig zurückgedrängt. Für eine strikte Kompetenzabgrenzung fehlt auch die übergreifende Klammer einer auf nationaler Souveränität beruhenden Verfassung, denn die europäischen Gerichte sind auf übernationalem Recht beruhende Vertragsgerichte. ${ }^{10}$ Sie stützen ihre Befugnisse allein auf einen Vertrag, dessen Herren die Mitgliedstaaten oder die Hohen Vertragsparteien bleiben.

\section{Die Grundrechte-Charta der EU und die Verfassungen der Mitgliedstaaten}

\section{Die Geltung der EU-Grundrechte}

Die Europäische Grundrechte-Charta ist an ihre eigenen Anwendungsbestimmungen gebunden. Die Charta gilt deshalb für die EU-Organe. ${ }^{11}$ Die Technik der Kompetenzabgrenzung funktioniert in diesem Bereich, weil sie auf das handelnde Organ abstellt und dadurch eine klare Zuweisung an das jeweilige Gericht erzielt.

11 Art. 51 Abs. 1 S. 1 EuGRCh. Vgl. z.B. E. Pache, in: Vedder/Heintschel von Heinegg (Hrsg.), Europäisches Unionsrecht, 2012, Art. 19, Rn. 8. 
In den Mitgliedstaaten sollen die EU-Grundrechte nach Art. 51 ff. GrundrechteCharta indessen nicht allgemein und umfassend gelten. Hier verzahnen sie sich mit den nationalen Grundrechten, denn sie sind ausdrücklich nur bei der Durchführung des Rechts der Union durch die Mitgliedstaaten anwendbar. Art. 51 Abs. 2 Grundrechte-Charta bestimmt sogar in einer - sonst selten zu findenden - negativen Kompetenzregel expressis verbis, dass die Charta den Geltungsbereich des Unionsrechts in keiner Weise ausdehne und keine neuen Zuständigkeiten begründe. Damit wirken unionale Grundrechte, wenn Mitgliedstaaten handeln, nur im Rahmen anderer EUVorschriften. Streitig ist allerdings der Begriff der „Durchführung des Rechts der Union" in den Mitgliedstaaten. In der Rechtssache Åkerberg Fransson ${ }^{12}$ hat sich der EuGH im Frühjahr weit vorgewagt und eine Geltung der Charta bereits behauptet, wenn sich im konkreten Fall irgendein Bezug zu einem Rechtssatz der EU ergeben könnte, genauer: solange ein Mitgliedstaat überhaupt ,im Anwendungsbereich des Unionsrechts"13 handele. Dann würden auch Vorschriften erfasst, welche gar nicht vom Mitgliedstaat als Verwaltungsaufgabe durchgesetzt werden, sondern in einem konkreten Fall nur als Erwägungspunkt in Betracht kommen könnten. Das Bundesverfassungsgericht hat daraufhin in seinem Urteil zum Antiterrordateigesetz ${ }^{14}$ einstimmig darauf hingewiesen, dass diese Ansicht weder mit dem Geist noch mit dem Wortlaut des Art. 51 Grundrechte-Charta oder mit Art. 6 Abs. 2 EU-Vertrag in Einklang zu bringen sei. Es geht davon aus, dass die Grundrechte-Charta erst dann angewendet werden kann, wenn das betroffene Rechtsverhältnis vom EU-Recht „determiniert“ 15 wird, d.h. für den konkreten Fall eine rechtlich verbindliche Anweisung vorgibt. Innerhalb dieser gelten die EU-Grundrechte, außerhalb davon sind sie nicht anzuwenden; eine Geltungserstreckung auf unional nicht verbindlich geregelte Bereiche würde die Rechtsprechungsbefugnisse des EuGH überschreiten und könnte vom Bundesverfassungsgericht nicht anerkannt werden. ${ }^{16}$

\section{Der Geltungsanspruch von EU- und Mitgliedstaatsgrundrechten im selben Fall}

Die Anwendung der EU-Grundrechte bei der Durchführung des Rechts der Union durch Mitgliedstaaten erweist sich aus einem zweiten Grund als regelmäßig problembeladen. Wenn ein Mitgliedstaat handelt, ist er stets an die eigene Verfassung gebunden. Er führt aber auch EU-Recht aus und muss die Chartarechte beachten. Hier überlagern sich immer zwei Grundrechtskomplexe. Wenn sie unterschiedliche Rechtsfolgen anordnen, kann wegen des Rangs und der Dignität der nationalen

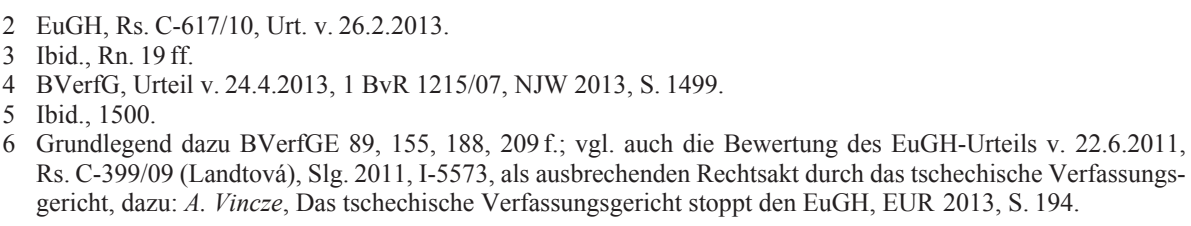


Grundrechte der Anwendungsvorrang des EU-Rechts nicht allgemein angenommen werden. ${ }^{17}$ Wo die mitgliedstaatlichen Rechte völlig ausgehebelt werden, verläuft sogar eine absolute Grenze für das Europarecht. ${ }^{18}$ Umgekehrt fordert die supranationale Rechtsordnung die europaweite Beachtung auch ihrer Grundrechte. Deren Ziel ist aber nicht die Herstellung identischer Grundrechtslagen oder gar deren Zentralisierung in Europa. Denn die Schutzbedürfnisse sind regional und national unterschiedlich. In diesen Fällen einer Grundrechtsüberlagerung ist die Methode der Kompetenzabgrenzung am Ende. Im Rechtsprechungsalltag muss eine andere Lösung gefunden werden, die beiden Grundrechtskomplexen genügt. Sie ist in Art. 51 Grundrechte-Charta auch bereits angelegt. Dort wird eine Geltung der unionalen Grundrechte angeordnet, aber nicht deren Vorrang. Die Autoren des Textes haben die Frage eines Anwendungsvorrangs eines der Grundrechtskomplexe offen gelassen. ${ }^{19}$ Auch beruht die Lehre vom Anwendungsvorrang allein auf der Rechtsprechung des EuGH. Anders als im sekundären Unionsrecht verlangen unionale Grundrechte aber überhaupt keinen Anwendungsvorrang, denn sie zielen auf optimalen Bürgerschutz, nicht auf Durchsetzung einer supranationalen Rechtsordnung. Das Motiv des effet utile verfolgt hier den Zweck der Grundrechtsoptimierung; führt also zum Vorrang des weiteren Grundrechts ohne Rücksicht auf dessen Herkunft. Eine Begründung unionalen Vorrangs, die sich auf dem Gedanken der implied power stützen wollte, stieße sich an der ausdrücklichen Schranke des Art. 51 Abs. 2 GG. Die in der EuGH-Rechtsprechung angeführte dogmatische Basis eines Anwendungsvorrangs trägt im Konflikt von nationalen und unionalen Grundrechten nicht.

Beide sollen im konkreten Fall gelten; das optimale Grundrecht sich im konkreten Fall durchsetzen. Das korrespondiert mit ihrer hohen Bedeutung auf der jeweiligen Ebene. Sie sind deshalb in Konkordanz zu bringen. Das Bundesverfassungsgericht bemüht sich im Dialog der Gerichte ${ }^{20}$ um eine solche Konkordanz, die beiden jeweils einschlägigen Grundrechten ihre Wirkung lässt. Es gibt erst an der äußersten Schwelle der Bedrohung der nationalen Verfassungsidentität ${ }^{21}$ und des wesentlichen Schutzgehalts von Grundrechten ${ }^{22}$ dem eigenen Grundgesetz den Vorrang. In diesen Extremfällen geht eine unmittelbar auf der Souveränität des Volkes beruhende Verfassung einem supranationalen, nur mittelbar demokratisch legitimierten Vertragsrecht vor. ${ }^{23}$

17 Vgl. zur Bedeutung der Grundrechte: J. Isensee, in: Merten/Papier (Hrsg.) (Fn. 7), § 26, Rn. 31 ff.

18 Vgl. zu den Vorbehalten mitgliedstaatlicher Verfassungsgerichte: D. Grimm, in: Merten/Papier (Hrsg.), Handbuch der Grundrechte, Band VI/2, 2009, § 168, Rn. $36 \mathrm{ff}$.

19 Vgl. D. Ehlers, in: ders., Europäische Grundrechte und Grundfreiheiten, 3. Aufl., 2009, § 14, Rn. 51.

20 F. C. Mayer, in: Grabitz/Hilf/Nettesheim (Hrsg.), Das Recht der Europäischen Union, 44. Ergänzungslieferung 2011, Art. 19, Rn. 43 f., 77 ff.; vgl. F. Kirchhof (Fn. 4), S. 3683 f., 3685.

21 BVerfGE 113, 273, 296; 123, 267, 353 f., 381 ff.; 126, 286, 302; vgl. auch A. Voßkuhle, Der europäische Verfassungsgerichtsverbund, NVwZ 2010, S. 1, $6 \mathrm{f}$.

22 BVerfGE 102, 147, 164.

23 BVerfGE 89, 155, 169; 123, 267, $364 \mathrm{ff}$. 


\section{Die Methode der Kooperation im Gerichtsverbund}

Zur Optimierung des Grundrechtsschutzes ist Kooperation zwischen beiden beteiligten Gerichten notwendig; deren Techniken heißen Zurückhaltung und Dialog zwischen den Gerichten, um beiden Grundrechtskomplexen gerecht zu werden.

\section{Die Technik der Zurückhaltung}

Die Technik der Zurückhaltung leitet beide Gerichte an, die eigene Zuständigkeit behutsam und mit Rücksicht auf das andere Gericht auszuüben. Das Bundesverfassungsgericht hat sie zum Beispiel in seinem ,Solange II“-Beschluss ${ }^{24}$ geübt, indem es seine eigene Rechtsprechung zurückgestellt hat, „solange“ der EuGH die deutschen Grundrechte im Wesentlichen in seiner Rechtsprechung achtet. Mit der neuen Grundrechte-Charta überlagern sich jetzt regelmäßig zwei Schutzrechte. Hier könnte zum Beispiel eine neue, umgekehrte „Solange“-Rechtsprechung des EuGH die notwendige Zurückhaltung wahren. Wo - wie in Deutschland - schon ein nationales Verfassungsgericht mit einer detaillierten Rechtsprechung vorhanden ist, könnte sich der EuGH in dieser Grauzone einer eigenen Chartarechtsprechung in Fällen aus diesem Mitgliedstaat enthalten, solange das nationale Gericht den Kerngehalt der EU-Grundrechte mit beachtet. Er müsste dann gar nicht vollständig auf die Auslegung und Durchsetzung unionaler Grundrechte verzichten, denn gegenüber den EU-Organen und anderen Mitgliedstaaten ohne Verfassungsgericht oder hinreichende Rechtsprechung würde er weiterhin tätig. Das nationale Verfassungsgericht würde seinerseits dann die europäischen Grundrechte in seine Erwägungen mit einbeziehen. Der Vorschlag belegt, dass es durchaus Mittel und Wege einer Kooperation durch Zurückhaltung gibt, welche die Freiheit des Bürgers optimiert. Sie würde zudem verhindern, dass der EuGH sich in seiner Rechtsprechungsarbeit übernimmt und nur punktuell Grundrechtsschutz gewährt. Denn der EuGH ist nicht als grundrechtschützendes Verfassungsgericht konzipiert. Bei ihm kann keine Individualverfassungsbeschwerde eingelegt werden; sie würde ihn personell auch sicherlich überfordern. Nationale Verfassungsgerichte, wie das Bundesverfassungsgericht, verfügen hingegen meistens über die Individualbeschwerde für den Bürger und sehen ihre Hauptaufgabe darin, mit ihrer Hilfe den Schutz von Bürger und Gesellschaft vor dem Zugriff einer mächtigen Staatsgewalt zu verwirklichen.

\section{Der Dialog im Gerichtsverbund}

Eine derartige „Solange“-Entscheidung des EuGH würde letztlich in einen Dialog beider Gerichte münden und zur zweiten Technik der Kooperation führen. Der so entstehende Rechtsprechungsverbund würde beide Gerichte anleiten, die Entscheidungen des jeweils anderen Spruchkörpers aufzufassen, dessen Argumentation zur 
Kenntnis zu nehmen und als Beitrag zum gemeinsamen Werk grundrechtsoptimierenden Bürgerschutzes in der eigenen Rechtsprechung $\mathrm{zu}$ berücksichtigen. Im Grundrechtsschutz am gleichen Strang ziehend, würden beide Gerichte eine gemeinsame Grundrechtsdogmatik im gegenseitigen Respekt vor der Auffassung des jeweils anderen Gerichts entwickeln können.

Ein vorsichtigeres Verfahren einer Kooperation durch den Dialog der Gerichte würden gegenseitige Anfragen an das andere Gericht im konkreten Fall sich überlappender Grundrechte bilden. So wie eine Vorlage nach Art. 267 AEUV dem europäischen Gericht die Gelegenheit einräumt, seine Rechtsauffassung zum europäischen Grundrecht kund zu tun, könnte es in einem laufenden Verfahren ein nationales Gericht um eine Stellungnahme zur Auslegung des betroffenen nationalen Grundrechts bitten. Der Sachverstand des jeweils Stellung nehmenden Gerichts würde im Prozess des anderen Gerichts zur Ausbildung einer gemeinsamen Grundrechtsjudikatur führen. Dass im europäischen Prozessrecht Anfragen beim nationalen Gericht nicht ausdrücklich vorgesehen sind, verbietet sie noch nicht; die Verfahrensgestaltung liegt im Ermessen des EuGH. Eine derartige Möglichkeit zur Einbringung der eigenen Expertise wird künftig sogar im Gerichtsverbund zwischen EGMR und EuGH zugunsten des letzteren vorgesehen, ${ }^{25}$ d.h. dort wird sie von der EU als förderlich angesehen. Warum sollte man sie dann zwischen EuGH und nationalen Verfassungsgerichten nicht auch praktizieren?

\section{Das Verhältnis des Europäischen Gerichtshofs für Menschenrechte zu den anderen Verfassungsgerichten}

\section{Die unterschiedliche Wirkung der Konvention auf die anderen Ebenen}

Komplizierter ist das Verhältnis des Europäischen Gerichtshofs für Menschenrechte in Straßburg zu den anderen Gerichten gestaltet. Während Art. 6 Abs. 2 und 3 EUVertrag eine weitgehende Verbindlichkeit der Urteile des EGMR für die Unionsorgane vorsieht, hängt die Wirkung seiner Urteile auf die Mitgliedstaaten von der jeweiligen Transformation der Konvention in die mitgliedstaatliche Rechtsordnung ab. In denjenigen Vertragsstaaten, in denen die EMRK unmittelbar gilt, entfalten die Entscheidungen des EGMR entsprechende Wirkung. Hier können die nationalen Verfassungsgerichte die Bestimmungen der EMRK sogar wie nationales Recht ebenfalls unmittelbar zum Schutze ihrer Bürger anwenden und auslegen. In anderen Staaten gilt die Konvention nur im Umfang des Parlamentsakts, der sie in innerstaatliches Recht transferiert hat. Dann entscheidet dieser Transformationsakt, wel-

25 Zum sog. Vorabbefassungsverfahren R. Uerpmann-Wittzack, Rechtsfragen und Rechtsfolgen des Beitritts der Europäischen Union zur EMRK, EuR-Beiheft 2012, S. 167, 170, 178 f.; vgl. Art. 3 Abs. 6 Report to the Committee of Ministers on the elaboration of legal instruments for the accession of the European Union to the European Convention on Human Rights v. 14.10.2011, Steering Committee for Human Rights (CDDH), Doc. CDDH(2011)009, abrufbar unter http://www.coe.int/t/dghl/standardsetting/hrpolicy/Accession/Meeting_repor ts/CDDH_2011_009_en.pdf. 
cher Rang der EMRK und den Entscheidungen des EGMR zukommt. In der Bundesrepublik Deutschland ist die Konvention zum Beispiel nach Art. 59 GG in die binnenstaatliche Rechtsordnung mit dem Rang eines einfachen Bundesgesetzes eingeführt worden. ${ }^{26}$ Damit erreicht sie nicht die Höhe des Verfassungsrechts, Grundgesetz und Bundesverfassungsgericht behalten die Vorhand. Die nationalen Fachgerichte haben die Konvention allerdings wie jedes andere Bundesgesetz zu beachten, wegen ihres menschenrechtlichen Inhalts überdies mit besonderer Berücksichtigung. ${ }^{27}$

\section{Die Lösung des Bundesverfassungsgerichts}

Hier wirkt sich das vom Bundesverfassungsgericht verfolgte Konzept eines Gerichtsverbunds geltungsfördernd für die Konvention aus. Da die Europäische Menschenrechtskonvention in 47 Vertragsstaaten ein Mindestniveau an Menschenrechten sichern soll, ${ }^{28}$ kann sie nicht wie jedes beliebige Bundesgesetz eingeordnet und angewendet werden. Das Bundesverfassungsgericht spricht ihr wegen ihres menschenrechtlichen Inhalts eine besondere Stellung auf der Ebene der einfachen Bundesgesetze $\mathrm{zu}^{29}$ und verlangt von den Fachgerichten, dass sie die Wertungen der Konvention beachten und die dort dem Staat aufgegebenen Pflichten erfüllen und durchsetzen, soweit es nach nationalem Verfassungsrecht möglich ist. ${ }^{30}$ Damit erlangt die Konvention nicht in der abstrakten Geltungsebene, aber in ihrer faktischen materiellen Bedeutung ein erhebliches Gewicht gegenüber dem einfachen Gesetzesrecht.

Das Bundesverfassungsgericht fügt sich in dieses Konzept des Gerichtsverbundes ein. Es lässt zum einen erneute Verfassungsbeschwerden ${ }^{31}$ über eine res iudicata zu, wenn mittlerweile neue Rechtsprechung des EGMR vorliegt, die menschenrechtlich zu anderen Ergebnissen führen würde. Für das Bundesverfassungsgericht wird damit der EGMR zum Dialogpartner, dessen Erwägungen und Argumente ihm zum erneuten Bewerten und Nachdenken veranlassen. Zugleich verlangt es vom EGMR unter Hinweis auf dessen Funktion, lediglich ein Mindestniveau an Menschenrechten in Europa, nicht aber einen flächendeckend hohen Grundrechtsstandard in allen Vertragsstaaten zu errichten, dass er sich gleichfalls in diesen Dialog einbindet ${ }^{32}$ und Wertungen des Bundesverfassungsgerichts in seine Prozesse aufnimmt. Der Gerichtsverbund zwischen Bundesverfassungsgericht und EGMR hat sich in dieser Hinsicht positiv entwickelt. Unser Gericht hat in mehreren spektaku-

26 Gesetz über die Konvention zum Schutze der Menschenrechte und Grundfreiheiten v. 7. 8. 1952, BGB1 II 1952 , 685; BVerfGE 124, 300, 319; 111, 307, 315 ff.; 74, 358, 370.

27 Vgl. BverfGE 111, 307, $324 \mathrm{f}$.

28 Vgl. D. Richter, in: Grote/Marauhn (Hrsg.) (Fn. 2), Kap. 9, Rn. 1 ff.

29 BVerfGE 74, 358, 370; 111, 307, 317.

30 BVerfGE, 111, 307, 327, 329; 128, 326, 371.

31 BVerfGE 128, 326, $364 \mathrm{f}$.

32 Grabenwarter/Pabel, Europäische Menschenrechtskonvention, 5. Aufl. 2012, §3, Rn. 11; vgl. F. Kirchhof (Fn. 4), S. $3681 \mathrm{f}$. 
lären Fällen eine andere Rechtsauffassung des Straßburger Gerichts aufgenommen und fortgeführt. ${ }^{33}$ Den EGMR haben nationale Besonderheiten im Grundrechtsschutz seiner Vertragsstaaten zunehmend zur Zurückhaltung in der eigenen Rechtsprechung unter der Rechtsfigur der margin of appreciation bewogen. ${ }^{34}$

\section{Die Kooperation bei regional besonderen Grundrechten}

\section{Seltenere Kollisionen}

Zuletzt ist das Thema Kooperation und Gerichtsverbund noch hinsichtlich der Grundrechte zu behandeln, die spezielle, regionale Besonderheiten bilden. Derartige Grundrechte finden sich häufig. Die Charta der europäischen Grundrechte weist zum Beispiel zahlreiche soziale Grundrechte ${ }^{35}$ sowie ausgedehnte und ausdifferenzierte Gleichheitsrechte ${ }^{36}$ auf, die in anderen Verfassungen wie dem deutschen Grundgesetz nicht zu finden sind. Für nationale Verfassungen gilt das Gleiche, denn deren Grundrechte sind aus historischen Erfahrungen des jeweiligen Staatsvolks entstanden. So wird zum Beispiel nach dem deutschen Grundgesetz die Pressefreiheit $^{37}$ sehr weitgehend geschützt, ist die Festlegung von Arbeitsbedingungen und Lohntarifen herkömmlich Arbeitgebern und Gewerkschaften überantwortet und dem Staat verschlossen ${ }^{38}$ oder werden den Beamten ${ }^{39}$ als einer besonderen Gruppe von Arbeitnehmern im öffentlichen Dienst besondere Grundrechte zugestanden. In Bundesstaaten existieren in den Ländern noch häufiger spezielle, regional bedingte Grundrechte. Die deutschen Landesverfassungen enthalten zum Beispiel eine Fülle von Grundrechten auf Heimat, ${ }^{40}$ auf bestimmte Ausbildungsstätten ${ }^{41}$ oder auf die Achtung und Förderung von Sport, Kultur und Minderheiten. ${ }^{42}$ Lediglich die Europäische Menschenrechtskonvention enthält keine besonderen Grundrechte. Das liegt auf der Hand, denn sie will nicht alle Grund-, sondern nur die universalen Menschenrechte als Mindeststandard gewährleisten und geht deshalb auf besondere Grundrechtsbedürfnisse anderer Provenienz nicht ein.

33 BVerfGE 92, 91, 107 ff. („,Feuerwehrabgabe“); BVerfGE 120, 180 („Caroline von Monaco III“); 128, 326 („,Sicherungsverwahrung II“); vgl. auch BVerfG NVwZ 2004, S. 852, 853 f.

34 EGMR, Urteil v. 7.12.1976 - 5493/72 (Handyside ./. Großbritannien), Z. 47 ff., vgl. Grabenwarter/Pabel, Europäische Menschenrechtskonvention, 5. Aufl. 2012, § 18, Rn. 20 f.; R. J. Schweizer, in: Merten/Papier (Hrsg.), Handbuch der Grundrechte, Band VI/1, 2010, § 138, Rn. $37 \mathrm{ff}$.

35 Insb. unter Titel IV. die Art. 27-38 GRCh; dazu S. Krebber, in: Callies/Ruffert (Hrsg.), EUV/AEUV, 4. Aufl. 2011, EU-GRCharta Art. 27, Rn. 2 ff.

36 Unter Titel III. etwa Art. Art. 20-26 GRCh.

37 Art. 5 Abs. 1 S. 2 GG; vgl. R. Grote/N. Wenzel, in: ders./Marauhn (Hrsg.) (Fn. 2), Kap. 18, Rn. 16 ff.; $U . D i$ Fabio, in: Maunz/Dürig, GG, 69. Ergänzungslieferung 2013, Art. 2, Rn. 235.

38 Art. 9 Abs. 3 GG.

39 Art. 33 Abs. 4, 5 GG.

40 Art. 2 Abs. 2 BWVerf; Art. 5 Abs. 1 S. 2 SächsVerf.

41 Vgl. etwa Art. 128, 164, 166 BayVerf; Art. 29 BbgVerf; Art. 106 SächsVerf.

42 Vgl. zur Sportförderung Art. 3c Abs. 1 BWVerf; Art. 32 BlnVerf; Art. 30 Abs. 3 ThürVerf; zur Kulturförderung zB. Art. 18 Abs. 1, 2 Verf NRW; Art. 34 SaarVerf; Art. 34 BbgVerf; und zum Minderheitenschutz Art. 5 SächsVerf; Art. 25 BbgVerf; Art. 5 Abs. 2 SHVerf; Art. 17 RhPfVerf. 
Bei diesen national oder regional besonderen Grundrechten wird es wegen ihrer jeweiligen Einzigartigkeit selten zu Kollisionen mit Entscheidungen von Gerichten aus anderen Ebenen kommen, denn sie geben nur zusätzlichen, erweiterten Schutz in besonderen Fällen. Ich möchte sie dennoch erwähnen, weil Gerichtsverbund und Grundrechtsdialog zwischen den Gerichte sich auch hier zeigen. Wer den Bürger grundrechtsoptimierend schützen will, muss auch auf seine besonderen Lagen eingehen. Die eher europa- oder nationalweit agierenden Gerichte vergessen oft die besonderen Gefährdungen oder historischen Erfahrungen regionaler Art. Hier sind in erster Linie im Bundesstaat die Landesverfassungsgerichte, in Zentralstaaten die nationalen Verfassungsgerichte gefordert, Grundrechtsschutz zu gewährleisten. Diese Grundrechte zeigen ein weiteres Mal die Notwendigkeit der Existenz von nationalen Verfassungsgerichten.

\section{Zurückhaltung auf den oberen Ebenen}

Für die anderen Grundrechts- und Gerichtsebenen gebieten sie eine Zurückhaltung in der Durchsetzung des eigenen Grundrechtskatalogs. Der Gerichtsverbund hält sie dazu an, nationale oder regionale Grundrechte auch im Normbereich des jeweils eigenen Grundrechtes zu berücksichtigen. Art. 52 Abs. 6 Grundrechte-Charta nimmt das auf, indem er anordnet, den einzelstaatlichen Rechtsvorschriften und Gepflogenheiten in vollem Umfang Rechnung zu tragen. ${ }^{43}$ Der EGMR zeigt sich für diese Frage sensibel, indem er nur einen Grundstandard an Menschenrechten fordert und bei deutlichen nationalen Unterschieden im Grundrechtsschutz die Rechtsfigur der margin of appreciation aktiviert. ${ }^{44}$

\section{Ausblick}

Kooperation und Gerichtsverbund werden mittlerweile zur wichtigen Aufgabe für alle beteiligten Verfassungs- und Vertragsgerichte. Zurückhaltung und Dialog sind unerlässlich, um den Grundrechtsschutz für den Bürger zu optimieren statt ihn zu zersplittern oder aufzuweichen. Sie sind arbeitsintensiv, weil sie fordern, auf das jeweils andere Gericht zu hören, fremde rechtliche Argumente aufzugreifen und sich in der eigenen Rechtsprechung zurückzuhalten. Der Gewinn eines gelungenen Dialogs und Verbunds liegt aber auf der Hand: Es entsteht ein Grundrechtsschutz in Europa, der über die gesamte Fläche Mindestschutz und Rechtssicherheit gewährt, ohne ziellos nur auf Vereinheitlichung oder Zentralität hinzuwirken und die nationalen und regionalen Besonderheiten zu übersehen. 